\section{Fibrodysplasia Ossificans Progressiva in a 5-Year Boy}

Sir,

Fibrodysplasia ossificans progressiva (FOP), also called stone man syndrome, and previously known as myositis ossificans progressiva, is an extremely rare mesodermal disorder (incidence of 1 per 2 million people) with autosomal dominant inheritance pattern; but most cases occur due to spontaneous mutation. ${ }^{1}$ It was first reported in 1736 by an English physician in a 14-year boy but the entity remained a mystery until $20^{\text {th }}$ Century. ${ }^{2}$ Patients with FOP are normal at the time of birth except deformity in great toes. ${ }^{3}$ These patients present with painful soft tissue swellings (flareups) at the age of 2 to 5 years, which later transform into calcifications, 4 leading to deformities and permanent dysmobility or immobility. ${ }^{5}$

Heterotopic calcification in FOP usually follows a systematic pattern and is seen first in dorsal, axial, cranial and proximal parts and then progresses to involve ventral, appendicular, caudal and distal regions. ${ }^{5}$ Unexpectedly, a few muscles are spared from the wrath of this disorder; these include diaphragm, tongue and extraocular muscles, smooth muscles and cardiac muscles. The deformity caused by FOP is so extreme that patients, who suffer from this wicked disorder, are usually bed and wheel-chair bound by the end of their 3 rd decade and develop a number of other problems like weight loss due to limited jaw movement, breathing problem due to the involvement of the ribs, and

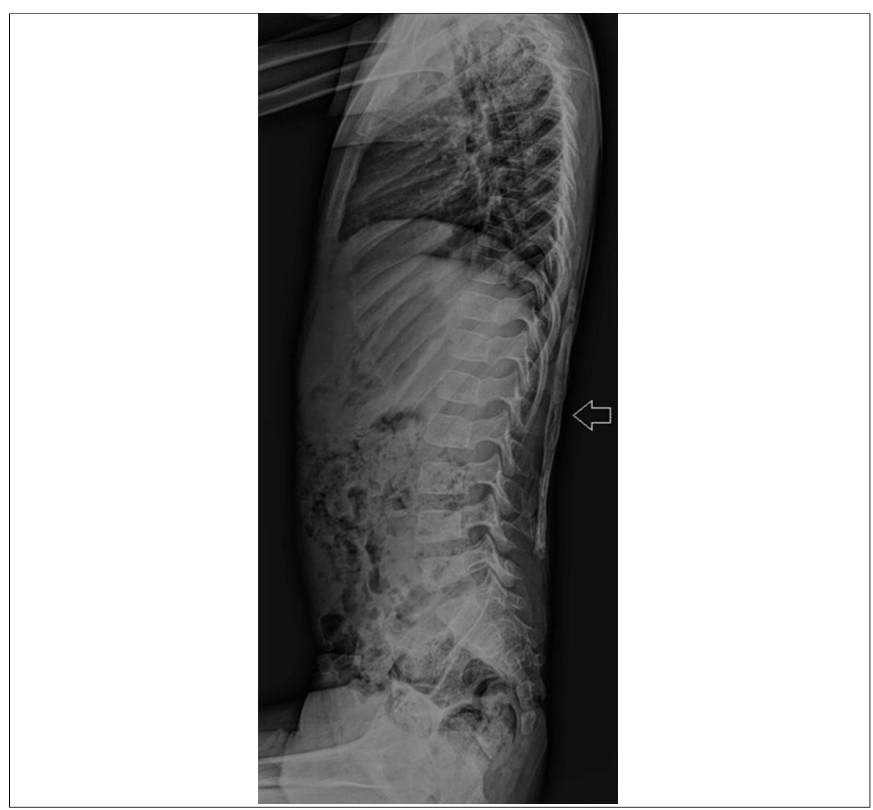

Figure 1a: X-ray dorsolumbar spine lateral view showing large sheet-like calcification within the soft tissues of back. decreased fertility. Furthermore, FOP tends to aggravate with surgery and minor trauma like bruises, falls, intramuscular injections etc. which results in the development of new flareups. ${ }^{6}$ Unfortunately, this entity is commonly misdiagnosed, resulting in catastrophic interventions like biopsies, which further exacerbate the disorder. ${ }^{5}$

A 5-year boy presented with history of painful swellings in the back, which increased over the period of a year or so. On examination, he had stony hard back and left scapular region painful swellings and deformed great toes. So, his X-ray was requested as a preliminary imaging tool which revealed large sheet-like calcifications within the soft tissues of back, which were more towards the right side of spine (Figure 1a). Further, linear calcifications were seen in the left scapular region and along the angle of right 6th and 7 th ribs (Figure $1 \mathrm{~b}$ ). X-ray of the feet revealed bilateral hallux valgus (Figure 2). Therefore, on the basis of clinical history and radiographic findings, diagnosis of FOP was made.

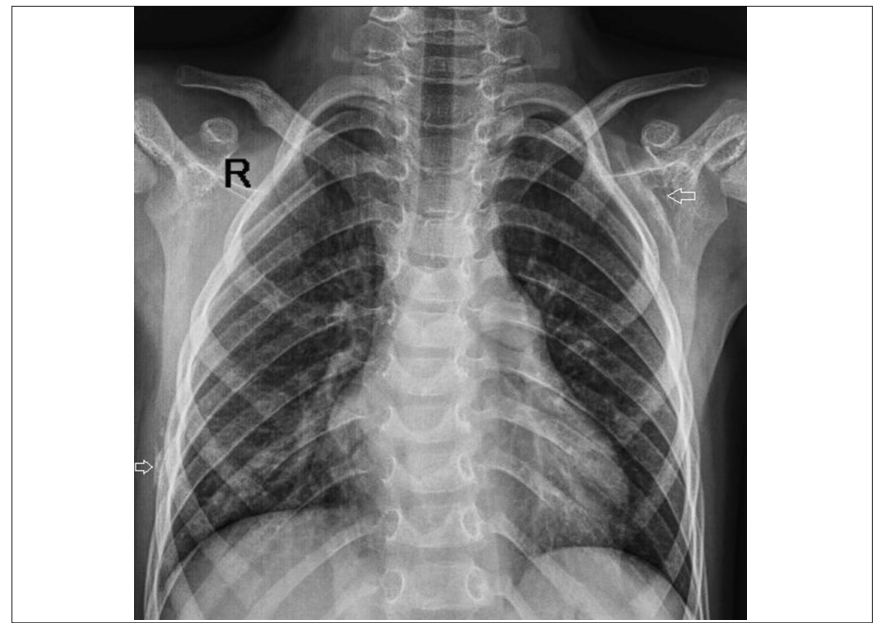

Figure 1b: X-ray chest PA view showing linear calcification in the left scapular region and along the angle of right 6 th and 7 th ribs.

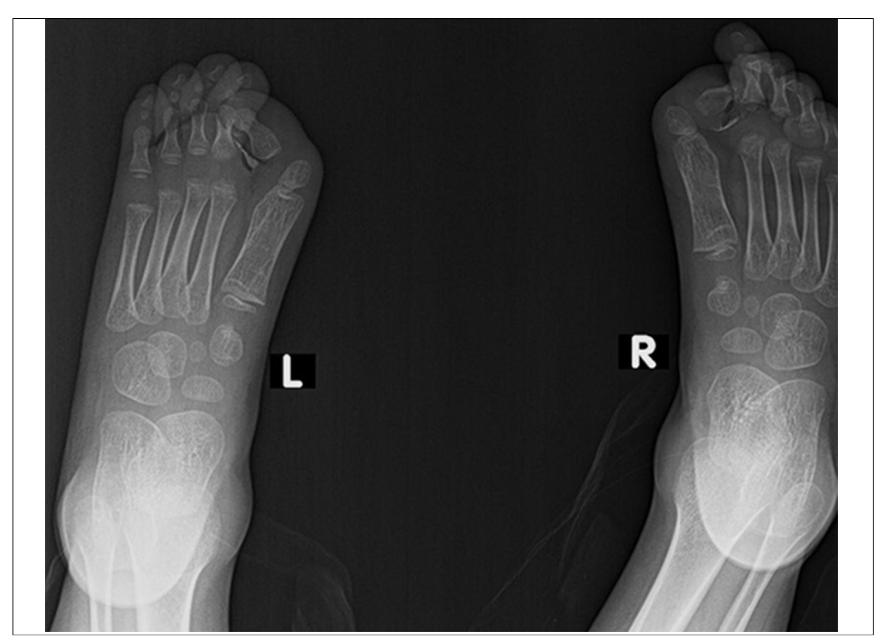

Figure 2: X-ray AP view of feet showing bilateral hallux valgus. 
FOP should be considered in cases where the patient presents with painful soft tissue swellings, which later become calcified and associated bilateral symmetrical great toe deformities. Knowledge of this rare entity is needed to prevent the misdiagnosis of this disorder which might lead to catastrophes.

\section{CONFLICT OF INTEREST:}

Authors declared no conflict of interest.

\section{AUTHORS' CONTRIBUTION:}

PAAA: Wrote the whole manuscript and participated in literature review.

IAP: Collected the patient data and images and participated in literature review.

\section{REFERENCES}

1. Deochand O, Anand N, Tarud RE, Su H. Fibrodysplasia ossificans progressiva: A case-based review. Poster ESSR 2017 / P-0243.

2. Kaplan FS. Fibrodysplasia ossificans progressiva: An historical perspective. Best Pract Res Clin Rheumatol 2005; 3:179-81.

3. Shore EM, Xu M, Feldman GJ, Fenstermacher DA, Cho TJ, Choi $\mathrm{IH}$, et al. A recurrent mutation in the BMP type I receptor ACVR1 causes inherited and sporadic fibrodysplasia ossificans progressiva. Nat Genet 2006; 38:525-7.
4. Cohen RB, Hahn GV, Tabas JA, Peeper J, Levitz CL, Sando A, et al. The natural history of heterotopic ossification in patients who have fibrodysplasia ossificans progressiva. J Bone Joint Surg Am 1993; 75:215-9.

5. Kaplan FS, Le Merrer M, Glaser DL, Pignolo RJ, Goldsby R, Kitterman JA, et al. Fibrodysplasia ossificans progressiva. Best Prac Res Clin Rheumatol 2008; 22:191-205.

6. Lanchoney TF, Cohen RB, Rocke DM, Zasloff MA, Kaplan FS. Permanent heterotopic ossification at the injection site after diphtheria-tetanus-pertussis immunizations in children who have fibrodysplasia ossificans progressiva. J Pediatr 1995; 126:762-4.

Pir Abdul Ahad Aziz' ${ }^{1}$ and Imtiaz Ali Panhwar ${ }^{2}$

1 Department of Radiology, Shaukat Khanum Memorial Cancer Hospital, Lahore, Pakistan

2 Department of Radiology, Liaquat National Hospital, Karachi, Pakistan

Correspondence to: Dr. Pir Abdul Ahad Aziz, Department of Radiology, Shaukat Khanum Memorial Cancer Hospital,

Lahore, Pakistan

E-mail: abdulahad.q@gmail.com

Received: November 24, 2018; Revised: January 30, 2019;

Accepted: February 01, 2019 